\title{
Risk Stratification Tools for Transient Ischemic Attack: Which Patients Require Hospital Admission?
}

Ethan Cumbler, MD Jeffrey J. Glasheen, mD
Hospital Medicine Group, Division of General Internal Medicine, Department of Medicine, University of Colorado, Denver School of Medicine, Denver, Colorado.

Stroke and transient ischemic attack (TIA) arise from identical etiologies and many fatal or disabling strokes are preceded by a TIA. Ten percent of patients presenting with a TIA will suffer a stroke within 3 months with half occurring in the first 48 hours. Still, many patients with a TIA do not receive timely evaluation or therapy. Hospitalization offers the opportunity for rapid evaluation and secondary prevention, reduced time to thrombolysis for early second strokes, and can be cost effective for high risk patients. Stratification tools are now available which allow individualized assessment of risk for early second strokes based on patient characteristics on presentation. The use of scoring systems such as the $\mathrm{ABCD}^{2}$ score to predict risk of stroke after TIA are useful in making an evidence-based judgment regarding need for hospitalization. High-risk patients have an $8.1 \%$ risk for stroke in the 48 hours after a TIA and warrant hospital admission. Intermediate-risk patients have a $4.1 \%$ risk of early second stroke and may be considered for admission, observation, or expedited clinic evaluation. Low-risk patients have a 2-day stroke risk of only $1 \%$ and are likely appropriate for prompt outpatient evaluation. TIA is a medical emergency, similar to unstable angina, and high risk patients should receive treatment and prevention measures instituted with comparable urgency. Journal of Hospital Medicine 2009;4:247-251. @ 2009 Society of Hospital Medicine.

KEYWORDS: clinical, evidence-based medicine, hospitalist, ischemic stroke, neurology, risk stratification, systems, transient ischemic attack.

Transient ischemic attacks (TIAs) are common and represent a clarion call to action to prevent disabling stroke. Incidence estimates for TIA range from 37 to 107 per 100,000 persons each year. ${ }^{1}$ Extrapolating from these data, there are likely greater than 100,000 to 300,000 TIAs in the US annually. Within 3 months, approximately $10 \%$ of these patients will suffer a stroke, with approximately one-half of these events occurring within the first 48 hours after the sentinel TIA. ${ }^{2-6}$ Nearly two-thirds of secondary strokes result in disability and $21 \%$ are fatal. ${ }^{3}$ Hospitalists are frequently called to provide care for patients with TIA and, as such, in order to establish an appropriate care plan, they require tools to better predict the likelihood and timing of a disabling stroke. $^{7}$ In this review we examine the rationale for early aggressive TIA evaluation and treatment in the hospital, overview risk stratification models to identify the patients at highest risk for early recurrent ischemia, and explore application of these tools to admission policy and individualized patient care planning.

\section{Definition}

TIA is defined as a brief episode of neurological dysfunction caused by focal brain or retinal ischemia with clinical symptoms typically lasting less than 1 hour and without evidence of brain infarction. ${ }^{8,9}$ Prior arbitrary time limits are being abandoned as advanced imaging techniques demonstrate that clinical examination lacks the sensitivity to detect small cerebral infarctions leading to misclassification of as many as $30 \%$ to $40 \%$ of strokes as TIAs. ${ }^{8-11}$ For cases in which imaging is not available, the diagnosis of "clinically probable
TIA" is suggested. Patients with imaging consistent with stroke appear to be at 4-fold to 10-fold higher risk for subsequent ischemic events, thus the presence of subclinical infarcts may have clinical importance., ${ }^{2,12}$ The majority of TIAs resolve within 1 hour of onset and neurologic deficit continuance beyond this time frame is more consistent with a stroke. ${ }^{13}$ Continuing symptoms after 1 hour mandates aggressive therapy in lieu of withholding intervention in the hopes of a spontaneous recovery.

\section{Rationale for Hospitalization}

Urgent evaluation and treatment within 24 to 48 hours of a TIA is recommended by the National Stroke Association (Table 1). ${ }^{14}$ These guidelines also recommend hospital admission for high-risk patients. There are a number of compelling arguments for the hospitalization of a patient at high risk for subsequent stroke.

First, hospitalization offers potential for reduced time to thrombolysis for those patients who have a second ischemic event in the early period following TIA. Outpatients with new ischemic stroke may see hours pass between symptom onset and presentation to the emergency department (ED). This delay frequently places them outside of the thrombolytic window. ${ }^{15-17}$ Hospitalization, assuming a well-designed inpatient stroke care system, has great potential to reduce this delay. Approximately $50 \%$ of the stroke risk following a TIA is evident within 48 hours and rapid thrombolysis, available in an inpatient setting, is associated with improved outcome after stroke. ${ }^{3,18}$ A cost-utility analysis found that a 24-hour admission for TIA patients to allow tissue 


\section{TABLE 1. Recommendations for the Initial Evaluation of Transient Ischemic Attack}

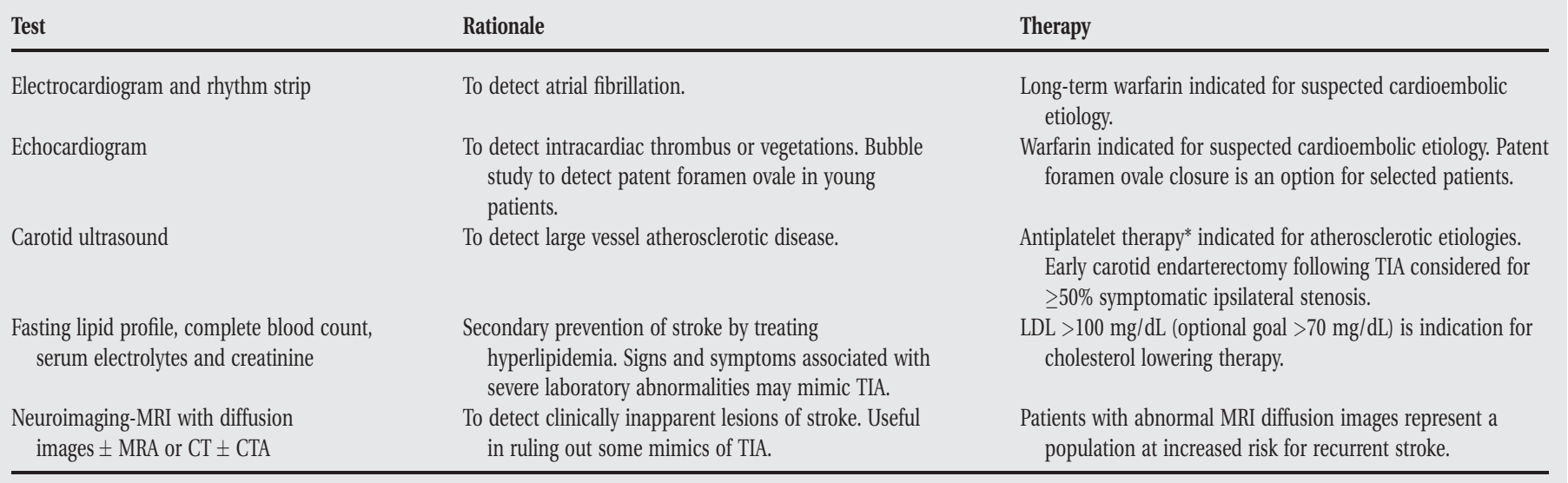

NOTE: Adapted from Johnston et al. ${ }^{14}$

Abbreviations: CT, computed tomography; CTA, computed tomographic angiography; LDL, low density lipoprotein; MRA, magnetic resonance angiography; MRI, magnetic resonance imaging.

*Aspirin/extended release dipyridamole or clopidogrel or aspirin.

plasminogen activator (t-PA) for recurrent ischemia has a cost-effectiveness ratio of $\$ 55,044$ per quality-adjusted life year with increasing cost effectiveness for the highest risk patients, such as those with a 24 -hour stroke risk of $>5 \% .{ }^{19}$

Second, hospital admission often facilitates the reliable and efficient evaluation for etiology and early initiation of secondary prevention. Neuroimaging, carotid ultrasound, echocardiography, and telemetry can be expedited with rapid initiation of proven secondary preventive therapies such as statin treatment, blood pressure control, and antithrombotic therapy. When indicated, carotid revascularization is recommended as soon as possible following TIA, with retrospective reviews suggesting improved outcomes when performed within 2 weeks of the event. ${ }^{14-20}$ In one analysis, a negative association between hospitalization for TIA and subsequent stroke was discovered by review of Canadian population-based administrative databases. ${ }^{5}$ While the mechanism for the negative association could not be established, the literature provides some support for hospitalization being associated with decreased risk for second strokes (hazard ratio [HR], 0.73; 95\% confidence interval [CI], 0.57-0.95). ${ }^{5}$

Theoretically, much of this evaluation and treatment could occur in the outpatient setting but delays commonly seen in outpatient evaluation and the high potential for early second strokes for some patients may make this a risky care plan. Despite the high likelihood for serious outcomes following TIA and clear guidelines for early evaluation and management, current care often lacks a sense of urgency. A 2004 Canadian study revealed that three-quarters of patients with a TIA were discharged directly from the ED with a resultant delay in diagnostic investigation. ${ }^{4}$ Over onethird of patients were discharged without a prescription for antithrombotic therapy. American primary care practice patterns reveal even more significant delays in therapy, with only $2 \%$ of patients admitted to a hospital on the day of presentation for TIA, despite $80 \%$ of patients presenting for evaluation on the day of symptom onset. ${ }^{21}$ In this study less than one-half of patients with atrial fibrillation were started on immediate anticoagulation. ${ }^{21}$ Further, as many as onethird of patients did not have any evaluation in the month after the index event. ${ }^{21}$ Hospitalization for high-risk patients has the potential to avoid these delays in outpatient evaluation and initiation of therapy.

Still, not all patients will require admission to a hospital setting. American EDs admit approximately one-half of all TIAs, with regional variability not explained exclusively by clinical characteristics. ${ }^{22}$ Focusing on identifying the cohort of patients who would most benefit from hospitalization is paramount. In general, hospitalization should be reserved for patients with higher risk of an early secondary stroke. Specifically, admission is generally recommended for patients with crescendo symptoms, TIA on antithrombotic therapy, or symptoms lasting $>1$ hour. ${ }^{14}$ Additionally, patients with symptomatic carotid stenosis of $\geq 50 \%$ and presumed cardioembolic or hypercoagulable etiology merit hospital admission. ${ }^{14}$ In many cases these etiologies may not be known at time of presentation. Evaluation, such as carotid ultrasound, may not be readily available in the ED to inform the admission decision. Several new scoring systems that utilize routine clinical features available within an hour of presentation have been developed to more objectively assess the risk of secondary stroke following a TIA. The use of these prognostic scoring systems is recommended by the National Stroke Association to aid in triaging this cohort of patients. ${ }^{14}$

\section{Prognostic Scoring Systems}

\section{California Score}

The 2000 California Score (Tables 2 and 3) is a 5-point tool retrospectively developed from a database of 1,707 


\section{TABLE 2. Risk Stratification Systems}

\begin{tabular}{|c|c|c|c|c|}
\hline \multicolumn{2}{|l|}{ Clinical Feature } & \multirow{2}{*}{$\begin{array}{l}\text { California Score (points) } \\
1\end{array}$} & \multirow{2}{*}{$\frac{\mathrm{ABCD} \text { (points) }}{1}$} & \multirow{2}{*}{$\frac{\mathrm{ABCD}^{2} \text { (points) }}{1}$} \\
\hline Age & $\geq 60$ years & & & \\
\hline Blood pressure & Systolic blood pressure $\geq 140$ or diastolic blood pressure $\geq 90 \mathrm{mmHg}$ & N/A & 1 & 1 \\
\hline Clinical deficits* & Unilateral weakness (focal motor weakness of 1 or more of face, arm, hand, or leg) & 1 & 2 & 2 \\
\hline & Speech impairment (dysarthria, dysphasia, or both) & 1 & 1 & 1 \\
\hline \multirow[t]{2}{*}{ Duration $^{\dagger}$} & $\geq 60$ minutes & 1 & 2 & 2 \\
\hline & 10-59 minutes & 1 & 1 & 1 \\
\hline Diabetes & Present & 1 & $\mathrm{~N} / \mathrm{A}$ & 1 \\
\hline Maximum score & & 5 & 6 & 7 \\
\hline
\end{tabular}

${ }^{*}$ California score allows 1 point for weakness and 1 point for speech impairment whereas the $A B C D$ and $A B C D{ }^{2}$ scores give 2 points for weakness or 1 point for speech impairment without weakness.

California score allows a maximum of 1 point for any duration $>10$ minutes whereas the $\mathrm{ABCD}$ and $\mathrm{ABCD}{ }^{2}$ allot 1 point for duration $10-59$ minutes or 2 points for duration $\geq 60$ minutes.

TIA patients seen in the ED of a California hospital system to predict the likelihood of stroke within 90 days of an initial presentation with transient neurologic deficits. ${ }^{3}$ Multivariate logistic regression models were used to test the clinical factors associated with stroke risk, resulting in a tool using clinical features of age, diabetes, symptom duration, and the type of deficit to provide quantitative estimates of intermediate term risk. Within 90 days, none of the patients with a score of zero had a stroke whereas the highest score had a $34 \%$ risk of stroke (Table 3). While it is possible that some patients with lower scores had a nonischemic etiology for their transient neurological symptoms, clinical practice contains similar ambiguity, and such patients would be correctly predicted to have a low risk for subsequent strokes. Additionally, the derivation and validation of this tool did not include a neurologist confirmation of TIA diagnosis; however, this likely mirrors the practice in most EDs. The California Score has subsequently been validated and expanded upon during the development of the $\mathrm{ABCD}^{2}$ score. $^{23}$ The California Score's utility is limited in the acute decision-making process, especially regarding the decision to admit, as it focuses on 90-day outcomes. For that, shorter-term risk assessments are more useful.

\section{ABCD Score}

The 2005 ABCD (Tables 2 and 4) is a 6-point tool designed to predict 7-day risk of stroke following TIA from the Oxfordshire Community Stroke Project, a UK prospective population-based cohort of 209 patients with diagnosis of TIA made by a neurologist. ${ }^{24}$ It evaluated factors previously found to be independent predictors of stroke after TIA, and determined that risk factors of age, blood pressure, type of clinical deficit, and symptom duration predicted 7 -day risk of stroke following TIA. Unlike the California score, the ABCD authors found presenting blood pressure, but not diabetes, to be independent predictors of future events. The authors validated the score with a second population of TIA patients in the Oxford Vascular Study and in a hospitalbased TIA clinic cohort. ${ }^{24}$ In the validation cohorts the score

\section{TABLE 3. California Score Predicted 90-Day Risk of Stroke Following TIA}

\begin{tabular}{lllllll} 
California Score & $\mathbf{0}$ & $\mathbf{1}$ & $\mathbf{2}$ & $\mathbf{3}$ & $\mathbf{4}$ & $\mathbf{5}$ \\
\hline 90-day stroke risk (\%) & 0 & 3 & 7 & 11 & 15 & 34 \\
\hline NOTE: Adapted from Johnston et al. $^{3}$ & & & & & \\
\hline
\end{tabular}

\section{TABLE 4. ABCD Score Predicted 7-Day Risk of Stroke} Following TIA

\begin{tabular}{lccccccc} 
ABCD Score & $\mathbf{0}$ & $\mathbf{1}$ & $\mathbf{2}$ & $\mathbf{3}$ & $\mathbf{4}$ & $\mathbf{5}$ & $\mathbf{6}$ \\
\hline 7-Day stroke risk (\%) & 0 & 0 & 0 & 0 & 2.2 & 16.3 & 35.5 \\
\hline NOTE: Adapted from Rothwell et al. & & & & & & \\
\hline
\end{tabular}

was highly predictive of stroke at 7 days $(P<0.001)$. Patients with the lowest scores of 0 to 3 had no strokes in the week following the index TIA, whereas patients with the highest score of 6 carried a 35.5\% risk of early second stroke. The primary limitation of the ABCD score lies in the small sample size, with only 18 recurrent strokes in the week after TIA in the derivation cohort.

The ABCD score has subsequently been tested in other settings with mixed results. Two studies found limited utility. $^{25,26}$ Other trials found high scores to be overly inclusive but highly predictive and thus the majority of evidence appears to support the validity of the ABCD score in predicting risk of early recurrent ischemic events. ${ }^{27-29}$ The ABCD score has been used to triage patients with high scores to inpatient management although the benefit of this strategy on outcomes has not been proven. ${ }^{30}$

\section{$A B C D^{2}$ Score}

The $2007 \mathrm{ABCD}^{2}$ (Tables 2 and 5) is a 7-point tool that uses the original $\mathrm{ABCD}$ criteria along with an additional point for 
TABLE 5. ABCD ${ }^{2}$ Score Predicted Risk of Stroke Following TIA

\begin{tabular}{llll} 
ABCD $^{2}$ Score & $\mathbf{0 - 3}$ & $\mathbf{4 - 5}$ & $\mathbf{6 - 7}$ \\
\hline Risk stratification & Low & Intermediate & High \\
2-Day stroke risk (\%) & 1 & 4.1 & 8.1 \\
7-Day stroke risk (\%) & 1.2 & 5.9 & 11.7 \\
90-Day stroke risk (\%) & 3.1 & 9.8 & 17.8 \\
\hline NOTE: Adapted from Johnston et al. ${ }^{23}$ & & & \\
\hline
\end{tabular}

diabetes. ${ }^{23}$ The $\mathrm{ABCD}^{2}$ derived a unified prognostic score for optimal prediction of the 2-day stroke risk from multivariate analysis of the original data sets used to create the California and ABCD scores. This score was then validated by the authors in 4 independent cohorts from the United States and the United Kingdom. ${ }^{23}$ In sum, 4809 patients with TIA were included in the $\mathrm{ABCD}^{2}$ analysis. Similar to prior studies, stroke occurred in $9.2 \%$ of patients by 90 days of which $20 \%$ were fatal. The authors created low (0-3 points), intermediate ( $4-5$ points), and high (6-7 points) risk categories. In the validation cohorts the prediction rule for the $\mathrm{ABCD}^{2}$ functioned better than the California or $\mathrm{ABCD}$ scores with c statistics of 0.62 to 0.83 (ideal prediction produces a c statistic of 1 and prediction no better than chance would have a c statistic of 0.5). They found a 2-day risk of stroke of $1 \%$ for low, $4.1 \%$ for intermediate, and $8.1 \%$ for the high risk group. Data from the study suggest $34 \%$ of TIA patients will be in low-risk, $45 \%$ in intermediate-risk, and $21 \%$ in high-risk categories. While the $\mathrm{ABCD}^{2}$ score overcomes some of the problems with the 2 prior systems it shares many of the limitations as it was derived from the combined data sets. All scoring systems lack the ability to provide guidance on the management of TIAs associated with rare conditions, such as vasculitis, that are underrepresented in the derivation data sets. The $\mathrm{ABCD}^{2}$ also does not incorporate imaging data and this will likely require further exploration.

The $\mathrm{ABCD}^{2}$ score can be used to predict risk for a variety of time intervals, has now been validated in independent Greek and British populations, and appears to be the best performing tool at predicting early risk of stroke regardless of underlying etiology. ${ }^{23,31,32}$ The authors suggest that admission for patients in the high-risk group is prudent whereas outpatient evaluation is reasonable for patients in the low-risk group. ${ }^{23}$ Admission for patients in the intermediate-risk group will depend on individualized decision making, local practice standards, and available community resources.

\section{New Models of Care: An Opportunity for Hospitalists}

The key to improving TIA outcomes appears to be more contingent on the speed of evaluation and initiation of appropriate therapy than on the location of the care. The EXPRESS trial studied the effect of an immediate access neurovascular clinic providing urgent evaluation and immediate treatment of nonhospitalized TIA patients versus usual care. Statistically significant reductions were seen in time to evaluation, first treatment prescription, and in 90-day risk of recurrent stroke $(10.3 \%$ versus $2.1 \%, P<0.0001)$ after the clinic was changed to the rapid evaluation and treatment model. $^{33}$

The SOS-TIA study used a 24-hour access hospital-based TIA clinic to evaluate the effects of rapid assessment and interventions on hospital length of stay and clinical outcomes. ${ }^{34}$ The 90 -day stroke rate was $1.24 \%$ (95\% CI, $0.71-$ 2.12 ), which represents a $79 \%$ reduction compared to the predicted stroke rate from the $\mathrm{ABCD}^{2}$ scores. With expedited evaluation and treatment, $74 \%$ of patients were able to be sent home on the same day.

The results of these 2 new studies provide compelling evidence that rapid evaluation and treatment in the first 48 hours after TIA has the potential to alter outcomes. Unfortunately not all communities have access to same day TIA clinics. Still, these findings should embolden hospitalists to advocate for urgent evaluation, such as neurology and cardiac imaging and carotid evaluation, with immediate initiation of secondary preventive therapy and early surgical intervention when appropriate. In most cases these changes will require process transformations that present prime opportunities for hospitalists to reengineer systems of care.

\section{Incorporating Prognostic Scores into Clinical Practice}

Applying the evidence to practice requires calculation of the early risk but also awareness of the community resources available. High-risk patients with an $\mathrm{ABCD}^{2}$ score of 6 or 7 have a very high $8.1 \%$ risk of stroke within the next 48 hours. Given the catastrophic outcomes frequently seen after second strokes, these patients warrant inpatient admission to facilitate the immediate initiation of appropriate secondary prevention and potentially shorten time to thrombolysis if an early stroke occurs. Intermediate-risk patients with $\mathrm{ABCD}^{2}$ scores of 4 and 5 have a $4.1 \%$ 2-day risk of stroke and may be considered for admission, hospital observation, or expedited clinic evaluation contingent on local availability. As many as one-third of TIA patients will be categorized as low risk with a score of 0 to 3 . These patients have a 2-day risk of stroke of only $1 \%$ and are likely safe for prompt outpatient evaluation and management. The new, validated, $\mathrm{ABCD}^{2}$ score is not a substitute for individualized judgment, but is helpful in developing admission guidelines in cooperation between neurologists, emergency room physicians, and hospitalists, and in using evidencebased medicine to provide optimal care for the patient presenting with a TIA.

Stroke and TIA arise from identical etiologies, respond to the same secondary preventive measures, and should be considered part of the spectrum of an ischemic cerebral syndrome. Recognizing TIA as a medical emergency with high rates of secondary stroke and subsequent disability 
allows institution of therapies with appropriate urgency. Hospitalization offers the ability to rapidly coordinate the testing and secondary prevention measures but also, for high-risk patients, offers the opportunity to reduce the time to thrombolysis for early recurrent strokes. New, validated scoring systems such as the $\mathrm{ABCD}^{2}$ score help the hospitalist to decide which patients are appropriate for admission and which can be managed in progressive and traditional outpatient settings.

\section{Address for correspondence and reprint requests:}

Dr. Ethan Cumbler, P.O. Box 6510 F782, 12605 E. 16th Ave, Aurora, CO 80045; Telephone: 720-848-4289; Fax: 720-848-4293;

E-mail: Ethan.Cumbler@ucdenver.edu Received 27 January 2008; revision received 15 June 2008; accepted 26 June 2008.

\section{References}

1. Ovbiagele B, Kidwell CS, Saver JL. Epidemiological impact in the United States of a tissue-based definition of transient ischemic attack. Stroke. 2003;34:919-924.

2. Douglas VC, Johnston CM, Elkins J, et al. Head computed tomography findings predict short-term stroke risk after transient ischemic attack. Stroke. 2003;34:2894-2899.

3. Johnston SC, Gress DR, Browner WS, et al. Short-term prognosis after emergency department diagnosis of TIA. JAMA. 2000;284:2901-2906.

4. Gladstone DJ, Kapral MK, Fang J, et al. Management and outcomes of transient ischemic attacks in Ontario. CMAJ. 2004;170:1099-1104.

5. Hill MD, Yiannakoulias N, Jeerakathil T, et al. The high risk of stroke immediately after transient ischemic attack: a population based study. Neurology. 2004;62:2015-2020.

6. Wu CM, McLaughlin K, Lorenzetti DL, et al. Early risk of stroke after transient ischemic attack: a systematic review and meta-analysis. Arch Intern Med. 2007;167:2417-2422.

7. Glasheen JJ, Epstein KR, Siegal E, et al. The spectrum of communitybased hospitalist practice: a call to tailor internal medicine residency training. Arch Intern Med. 2007;167:727-729.

8. Caplan LR. Transient ischemic attack with abnormal diffusion-weighted imaging results: what's in a name? Arch Neurol. 2007;64:1080-1082.

9. Hadjiev DI, Mineva PP. A reappraisal of the definition and pathophysiology of the transient ischemic attack. Med Sci Monit. 2007;13:RA50-53.

10. Boulanger J, Coutts SB, Eliasziw M, et al. Diffusion-weighted imagingnegative patients with transient ischemic attack are at risk of recurrent transient events. Stroke. 2007;38:2367-2369.

11. Albers GW, Caplan LR, Easton JD, et al. Transient ischemic attack-proposal for a new definition. $N$ Engl J Med. 2002;347:1713-1716.

12. Calvet D, Lamy C, Touze E, et al. Management and outcome of patients with transient ischemic attack admitted to a stroke unit. Cerebrovasc Dis. 2007;24:80-85.

13. Levy DE. How transient are transient ischemic attacks. Neurology. 1988; 38:674-677.

14. Johnston SC, Nguyen-Huynh MN, Schwarz ME, et al. National Stroke Association guidelines for the management of transient ischemic attacks. Ann Neurol. 2006;60:301-313.

15. Rossnagel K, Jungehulsing JG, Nolte $\mathrm{CH}$, et al. Out-of-hospital delays in patients with acute stroke. Ann Emerg Med. 2004;44:476-483.
16. Wester P, Radberg J, Lundgren B, et al. Factors associated with delayed admission to hospital and in-hospital delays in acute stroke and TIA: a prospective multicenter study. Stroke. 1999;30:40-48.

17. Morris DL, Rosamond W, Madden K, et al. Prehospital and emergency department delays after acute stroke: the Genentech Stroke Presentation Survey. Stroke. 2000;31:2585-2590.

18. Tissue plasminogen activator for acute ischemic stroke. The National Institute of Neurological Disorders and Stroke rt-PA Stroke Study Group. N Engl J Med. 1995;333:1581-1587.

19. Nguyen-Huynh MN, Johnston SC. Is hospitalization after TIA cost effective on the basis of treatment with tPA? Neurology. 2005;65:1799-1801.

20. Rothwell PM, Eliasziw M, Gutnikov SA, et al. Endarterectomy for symptomatic carotid stenosis in relation to clinical subgroups and timing of surgery. Lancet. 2004;363:915-924.

21. Goldstein LB, Bian J, Samsa GP, et al. New transient ischemic attack and stroke: outpatient management by primary care physicians. Arch Intern Med. 2000;160:2941-2946.

22. Coben JH, Owens PL, Steiner CA, et al. Hospital and demographic influences on the disposition of transient ischemic attack. Acad Emerg Med. 2008;15:171-176.

23. Johnston SC, Rothwell PM, Nguyen-Huynh MN, et al. Validation and refinement of scores to predict very early stroke risk after transient ischaemic attack. Lancet. 2007;369:283-292.

24. Rothwell PM, Giles MF, Flossmann E, et al. A simple score (ABCD) to identify individuals at high early risk of stroke after transient ischaemic attack. Lancet. 2005;366:29-36.

25. Cucchiara BL, Messe SR, Taylor RA, et al. Is the ABCD score useful for risk stratification of patients with acute transient ischemic attack? Stroke. 2006;37:1710-1714.

26. Purroy F, Molina CA, Montaner J, et al. Absence of usefulness of ABCD score in the early risk of recurrent stroke in transient ischemic attack patients. Stroke. 2007;38:855-856.

27. Tsivgoulis G, Spengos K, Manta P, et al. Validation of the ABCD score in identifying individuals at high early risk of stroke after a transient ischemic attack: a hospital-based case series study. Stroke. 2006;37: 2892-2897.

28. Sciolla R, Melis F. Rapid identification of high-risk transient ischemic attacks: prospective validation of the ABCD score. Stroke. 2008;39: 297-302.

29. Bray JE, Coughlan K, Bladin C. Can the ABCD score be dichotomized to identify high-risk patients with transient ischaemic attack in the emergency department? Emerg Med J. 2007;24:92-95.

30. Byrne A, Daly C, Rocke L, et al. Can risk stratification of transient ischaemic attacks improve patient care in the emergency department? Emerg Med J. 2007;24:637-640.

31. Selvarajah JR, Smith CJ, Hulme S, et al. Prognosis in patients with transient ischaemic attack (TIA) and minor stroke attending TIA services in the north west of England: The NORTHSTAR Study. J Neurol Neurosurg Psychiatry. 2007:1-6.

32. Tsivgoulis G, Vassilopoulou S, Spengos K. Potential applicability of the $\mathrm{ABCD}^{2}$ in triaging TIA patients. Lancet. 2007;369:1082.

33. Rothwell PM, Giles MF, Chandratheva A, et al. Effect of urgent treatment of transient ischemic attack and minor stroke on early recurrent stroke (EXPRESS Study): a prospective population-based sequential comparison. Lancet. 2007;370;1432-1442.

34. Lavallee PC, Meseguer E, Abboud H, et al. A transient ischemic attack clinic with round-the-clock access (SOS-TIA): feasibility and effects. Lancet Neurol. 2007;6:953-960. 